\title{
Construction Quality Evaluation of Teacher Development Schools Based on Improved Artificial Neural Network
}

\author{
https://doi.org/10.3991/ijet.v16i08.22129 \\ Xiaoyu Hua \\ Ningbo Childhood Education College, Ningbo, China \\ 2000005 encec.edu.cn
}

\begin{abstract}
There are many problems with the convention teacher education model, such as the short internship time during pre-job training, and the limited experience, pertinence, and effectiveness of on-the-job training. Fortunately, the teacher development school mechanism provides a viable solution to these problems. Therefore, the construction quality of such schools is of great significance to the teaching level and professional development of school teachers, as well as the overall development of students. As a result, this paper proposes a method to evaluate the construction quality of teacher development schools based on an improved artificial neural network. Firstly, an evaluation index system was established for the construction quality of teacher development schools, which consists of 5 core evaluation indices, and the periodical scoring criteria were detailed. Then, the feasibility of the proposed evaluation index system was verified through reliability, validity, and difference analyses. Finally, a combined neural network was constructed to evaluate the construction quality of teacher development schools. The experimental results show that our model can effectively predict the construction quality of teacher development schools, providing a reference for project quality evaluation in other fields.
\end{abstract}

Keywords-Artificial neural network, teacher development school, construction quality evaluation

\section{Introduction}

There are many problems with the convention teacher education model, such as the short internship time during pre-job training, and the limited experience, pertinence, and effectiveness of on-the-job training [1-4]. In order to produce high-quality professional teachers, countries around the world have made a lot of attempts to reform the conventional teacher education model. Gradually, a teacher development school mechanism that integrates training of teachers at different stages like pre-job, on-boarding and on-the-job periods [5-7] has been formed, which is of great significance to promoting the professional development of teachers and driving the overall development of students.

The practical experience gained by professional development schools (PDS) in the U.S. in pre-job training and post-job study of teachers has provided some reference for 
the theoretical research on teacher education model optimization and construction of teacher development schools [8-12]. Blasie and Palladino [13] established teacher education relationships for elementary and secondary schools and universities. With a secondary school in Kentucky Secondary School and the University of Texas at Austin as the examples, the paper conducted empirical analysis on the cooperation models, processes and effectiveness of teacher education in elementary and secondary schools and universities. Empirical analysis. Hixon and Buckenmeyer [14] conducted case analysis to evaluate Stanford University's pre-job teacher education program STEP from two aspects in practice - problem solving and countermeasures, and elaborates the reform plan for the PDS pre-job education practice system in the U.S., which mainly consists of educational organization management, collaborative operation mechanism, project implementation status and practical reform support. Sweeney et al. [15] reviewed the construction standards for exemplary teacher development schools, and proposed a teacher education model where "exemplary teacher development schools work as the guide, primary and secondary schools play the main roles and primary and secondary schools and universities collaborate with each other". Through examples, it also quantified the construction evaluation indices for general development schools and evaluated their construction status. Mitchell et al. [16] studied the operating mechanism of teacher development schools at the county level. With a teacher development school in Beijing as the example, it analyzed the internal and external factors affecting its development, and also proposed improvement strategies from the perspectives of functional structure, operational mode and operational support considering the difficulties encountered in school construction. Lin et al. [17] divided the construction models of teacher development schools into discipline-driven and expert-governed ones, and proposed adopting cooperation activities such as "teacher development research course" and "professional teacher development forum" to reduce the differences in campus cultures between universities and primary and secondary schools, enhance mutual needs for cooperation and maintain the sustainable development of cooperation. The professional development of teachers is the key to the new curriculum reform of elementary education. Oostendorp et al. [18] connected education theories with elementary and secondary school education practices, and proposed a deeply involving cooperation approach that can change teachers' thoughts and behaviors, which provided professional support for the education practices of primary and secondary school teachers on the specific operational level.

The existing research on teacher development schools mostly focuses on comparing their characteristics, summarizing the guiding roles of training and verifying the operating mechanism [19-23]. Only a few papers mentioned the evaluation of school construction quality, and even in case analysis, there has been very little discussion on the school construction quality evaluation. To this end, this paper proposes a construction quality evaluation method for teacher development schools based on an improved artificial neural network. Section 2 of this paper constructs an evaluation index system for the construction quality of teacher development schools, which consists of 5 core evaluation indices, and innovatively sets up periodical scoring criteria for each secondary index. Section 3 analyzes the feasibility of the evaluation system for the construction quality of teacher development schools from three aspects - reliability, validity and 
difference. Section 4 builds a predictive model for evaluating the construction quality of teacher development schools based on the unsupervised self-organizing feature map neural network and the supervised BP neural network. The experimental results prove that the model can deliver effective prediction.

\section{Establishment of the Evaluation Index System for the Construction Quality of Teacher Development Schools and Analysis of Scoring Criteria}

Table 1. The evaluation index system for the construction quality of teacher development schools

\begin{tabular}{|c|c|c|}
\hline \multicolumn{2}{|r|}{ Evaluation indices for the construction quality of teacher development schools } & No. \\
\hline \multirow{4}{*}{$\begin{array}{l}\text { Learning } \\
\text { group A1 }\end{array}$} & $\begin{array}{l}\text { Providing a comprehensive learning environment that supports multiple types of } \\
\text { learners }\end{array}$ & B1 \\
\hline & Conducting exploratory research and practice for learning & $\mathrm{B} 2$ \\
\hline & $\begin{array}{l}\text { Making supplements to and updating the research and practical results on the teaching } \\
\text { and learning of teachers and students }\end{array}$ & B3 \\
\hline & Expanding learner groups or partner institutions in a timely manner & B4 \\
\hline \multirow{5}{*}{$\begin{array}{l}\text { Training } \\
\text { quality den- } \\
\text { sity } \\
\text { A2 }\end{array}$} & Providing timely and professional evaluation of learning tasks, goals and effects & B5 \\
\hline & Providing regular summary and announcements of learning results & B6 \\
\hline & Applying professional and consistent standards & B7 \\
\hline & $\begin{array}{l}\text { Giving cyclical evaluation, feedback, summary and update of research and practical } \\
\text { results }\end{array}$ & B8 \\
\hline & $\begin{array}{l}\text { Continuously communicating with local governments, universities, specialized entities } \\
\text { and scientific research organizations on training targets }\end{array}$ & B9 \\
\hline \multirow{3}{*}{$\begin{array}{l}\text { Organiza- } \\
\text { tional control } \\
\text { function A3 }\end{array}$} & Having blurred boundaries of cooperation and unified training targets & $\mathrm{B} 10$ \\
\hline & $\begin{array}{l}\text { Having equal principles for roles and resources allocation and a regulated reward and } \\
\text { punishment mechanism }\end{array}$ & B11 \\
\hline & Maintaining continuous optimization of the cooperation environment & B12 \\
\hline \multirow{3}{*}{ Fairness A4 } & Learning opportunities are not affected by personal subjective factors or achievements & B13 \\
\hline & \begin{tabular}{|l|l} 
Diverse approaches to learning \\
\end{tabular} & B14 \\
\hline & Having professional and consistent evaluation criteria for courses and systems & B15 \\
\hline \multirow{5}{*}{$\begin{array}{l}\text { Process man- } \\
\text { agement A5 }\end{array}$} & $\begin{array}{l}\text { Updating the management structure with the expansion of members, and maintaining } \\
\text { consistency in values and culture }\end{array}$ & B16 \\
\hline & $\begin{array}{l}\text { Paying constant attention to and evaluating the training targets and the current training } \\
\text { status }\end{array}$ & B17 \\
\hline & $\begin{array}{l}\text { Setting the permissions of different roles and establishing the reward and punishment } \\
\text { system }\end{array}$ & B18 \\
\hline & \begin{tabular}{|l|} 
Having a sound resources sharing system \\
\end{tabular} & B19 \\
\hline & Having rich resource support & $\mathrm{B} 20$ \\
\hline
\end{tabular}

Based on the understanding of the standards for and elements of teacher development schools and by referring to the standard philosophies and evaluation procedures for PDS, this paper constructed an evaluation index system for the construction quality of teacher development schools, as shown in Table 1. The system consists of 5 core evaluation indices, namely learning group A1, training quality density A2, organizational control function A3, fairness A4 and process management A5. This paper believes that 
the construction of a scientific teacher development school begins with adequate preparations, including carrying out inquisitive research and practice of learning organizations, making the school students-centred, training teachers and students in practice and also adhering to training goals, values and cultures and maintaining professional and consistent evaluation criteria for curriculums and systems. In the construction process, it is necessary to actively develop new roles and relationships, blur the boundaries of cooperation, focus on resources allocation and sharing and maintain and timely expand the learner groups and major partners.

Table 2. Periodical scoring criteria for sub-indices

\begin{tabular}{|c|c|c|c|}
\hline No. & Periodical scoring criteria & No. & Periodical scoring criteria \\
\hline B1 & $\begin{array}{l}\text { Creation } \rightarrow \text { support } \rightarrow \text { diversity } \rightarrow \text { ap- } \\
\text { plication }\end{array}$ & B11 & $\begin{array}{l}\text { Developing new roles } \rightarrow \text { redistributing resources } \\
\rightarrow \text { assigning equal rights and interests } \rightarrow \text { reforming } \\
\text { the roles and resources structure }\end{array}$ \\
\hline B2 & $\begin{array}{l}\text { Sharing } \rightarrow \text { partial application } \rightarrow \text { practice } \\
\rightarrow \text { continuation and sharing }\end{array}$ & B12 & $\begin{array}{l}\text { Reflecting the values of participants } \rightarrow \text { showing re- } \\
\text { spect and reliance } \rightarrow \text { maintaining the cooperative } \\
\text { relations } \rightarrow \text { normalizing cooperation }\end{array}$ \\
\hline B3 & $\begin{array}{l}\text { Supporting practice } \rightarrow \text { receiving evalua- } \\
\text { tion } \rightarrow \text { selecting the optimal one } \rightarrow \text { feed- } \\
\text { back and correction }\end{array}$ & B13 & $\begin{array}{l}\text { Paying attention to fairness } \rightarrow \text { studying subjective } \\
\text { differences } \rightarrow \text { proving the existence of differences } \\
\rightarrow \text { reducing differences }\end{array}$ \\
\hline B4 & $\begin{array}{l}\text { Planning expansion } \rightarrow \text { participants ex- } \\
\text { pansion } \rightarrow \text { partner expansion } \rightarrow \text { learning } \\
\text { group expansion }\end{array}$ & B14 & $\begin{array}{l}\text { Learner type identification } \rightarrow \text { personal learning } \\
\text { strategy customization } \rightarrow \text { effectiveness verification } \\
\rightarrow \text { application }\end{array}$ \\
\hline B5 & $\begin{array}{l}\text { Unifying evaluation criteria } \rightarrow \text { consider- } \\
\text { ing actual reform } \rightarrow \text { verification } \rightarrow \text { eval- } \\
\text { uation decision making }\end{array}$ & B15 & $\begin{array}{l}\text { Standard diversification } \rightarrow \text { effectiveness verifica- } \\
\text { tion } \rightarrow \text { system verification } \rightarrow \text { results display }\end{array}$ \\
\hline B6 & $\begin{array}{l}\text { Evaluation by teachers and students } \rightarrow \\
\text { results report } \rightarrow \text { results announcement } \\
\rightarrow \text { results discussion }\end{array}$ & B16 & $\begin{array}{l}\text { Approving } \rightarrow \text { planning } \rightarrow \text { unifying core values and } \\
\text { culture } \rightarrow \text { multiple parties participating }\end{array}$ \\
\hline B7 & $\begin{array}{l}\text { Discussing standards } \rightarrow \text { setting standards } \\
\rightarrow \text { disclosing standards } \rightarrow \text { verifying } \\
\text { standard }\end{array}$ & B17 & $\begin{array}{l}\text { Paying attention } \rightarrow \text { evaluating effectiveness } \rightarrow \text { es- } \\
\text { tablishing an evaluation mechanism } \rightarrow \text { conducting } \\
\text { regular review }\end{array}$ \\
\hline B8 & $\begin{array}{l}\text { Paying attention to evaluation } \rightarrow \text { con- } \\
\text { ducting evaluation } \rightarrow \text { giving evaluation } \\
\text { feedbacks } \rightarrow \text { carrying out continuous cy- } \\
\text { clical evaluation }\end{array}$ & B18 & $\begin{array}{l}\text { New job opportunities emerging } \rightarrow \text { new roles ap- } \\
\text { pearing } \rightarrow \text { setting existing role permissions } \rightarrow \text { car- } \\
\text { rying out continuous identification }\end{array}$ \\
\hline B9 & $\begin{array}{l}\text { Supporting and restraining explora- } \\
\text { tion } \rightarrow \text { developing relationships } \rightarrow \text { carry- } \\
\text { ing out continuous dialogues } \rightarrow \text { influenc- } \\
\text { ing the process }\end{array}$ & B19 & $\begin{array}{l}\text { Analyzing resources } \rightarrow \text { making commitments on } \\
\text { sharing } \rightarrow \text { providing resources } \rightarrow \text { maintaining re- } \\
\text { sources sharing }\end{array}$ \\
\hline B10 & $\begin{array}{l}\text { Planning cooperation } \rightarrow \text { carrying out co- } \\
\text { operation } \rightarrow \text { promoting the improvement } \\
\text { of training quality } \rightarrow \text { expanding the } \\
\text { scope of cooperation }\end{array}$ & B20 & $\begin{array}{l}\text { Establishing a communication mechanism } \rightarrow \text { carry- } \\
\text { ing out effective communication } \rightarrow \text { expanding the } \\
\text { scope of communication } \rightarrow \text { carrying out multi-fac- } \\
\text { eted communication }\end{array}$ \\
\hline
\end{tabular}

The evaluation standards for the construction quality of teacher development schools should be applicable to different situations at different development stages. Therefore, the scoring criteria for the 20 sub-indices under the 5 core evaluation indices should be established by stage according to the construction status of the schools. This paper defined four development stages for each sub-index, namely the initial stage, the development stage, the maintenance stage and the influence stage. Each development stage 
of each sub-index corresponds to a different planning direction, degree of consistency, construction level, degree of institutionalization, and status of support, so there are also differences in the quantitative scores. In this way, scores are given to the 20 sub-indices according to the staged construction level, which not only avoids the shortcomings when a single index is used to define the construction quality, but also truly reflects the construction process and progress of a teacher development school.

\section{Feasibility Analysis on the Construction Quality Evaluation of Teacher Development Schools}

Reliability and validity are important standards for quality testing. Table 3 shows the results of the adequacy and Mauchly's test of sphericity in SPSS with respect to the construction quality evaluation of a teacher development school, that is, the results of the investigation on whether the evaluation indices mentioned above are suitable for factor analysis. The adequacy value of the construction quality evaluation of the teacher development school is 0.787 , showing that the evaluation indices in the evaluation index system provided in the previous section have common factors, so the following factor analysis can be performed: take 20 evaluation sub-indices as 20 variables and the 5 core indices for evaluation of teacher development school construction as 5 factors; calculate the correlation coefficients of the 20 evaluation sub-indices and group them based on the principal component analysis method. Through the test, it was found that the grouping results of the 20 evaluation sub-indices basically conformed to the preset quantitative results of the evaluation index system and that there were negative items, indicating that the evaluation index system for the construction quality of teacher development schools is effective.

In terms of reliability check, this paper conducted an internal consistency check on the 5 core evaluation indices. Table 4 shows the test result - the internal consistency coefficient value is 0.721 , indicating that the core evaluation indices used in this paper are also highly reliable.

In order to further evaluate the evaluation values of the 5 core evaluation indices, this paper conducted a correlation analysis of the indices. The Pearson correlation analysis results are shown in Table 5. It can be seen that the 5 core evaluation indices are significantly correlated with the overall construction quality of a teacher development school, and the correlation coefficients are all greater than 0.5 , indicating that the 5 core evaluation indices are complementary to and dependent on each other and are all key components of the construction quality evaluation of teacher development schools. 
Table 3. Adequacy and Mauchly's test of sphericity in SPSS with respect to the construction quality evaluation of a teacher development school

\begin{tabular}{|l|l|c|}
\hline \multicolumn{2}{|c|}{ Adequacy check } & $\mathbf{0 . 7 8 7}$ \\
\hline \multirow{3}{*}{ Mauchly's test of sphericity in SPSS } & Degree of freedom & 611 \\
\cline { 2 - 3 } & Significance & 0.001 \\
\cline { 2 - 3 } & Chi-squared approximation & 1254.51 \\
\hline
\end{tabular}

Table 4. Internal consistency check on the tiered evaluation of teacher development school construction quality

\begin{tabular}{|l|c|c|c|}
\hline \multicolumn{2}{|c|}{ Dimension } & Reliability check & Number of items \\
\hline \multirow{4}{*}{ Core evaluation indices } & $\mathrm{A} 1$ & 0.566 & 8 \\
\cline { 2 - 4 } & $\mathrm{A} 2$ & 0.611 & 10 \\
\cline { 2 - 4 } & $\mathrm{A} 3$ & 0.528 & 6 \\
\cline { 2 - 4 } & $\mathrm{A} 4$ & 0.479 & 6 \\
\cline { 2 - 4 } & $\mathrm{A} 5$ & 0.519 & 10 \\
\hline $\begin{array}{l}\text { Overall level of the construction quality evaluation of a teacher } \\
\text { development school }\end{array}$ & 0.721 & 40 \\
\hline
\end{tabular}

Table 5. Correlation analysis on the primary construction quality evaluation of a teacher development school

\begin{tabular}{|c|c|c|c|c|c|}
\hline & $\mathbf{A 1}$ & $\mathbf{A 2}$ & $\mathbf{A 3}$ & $\mathbf{A 4}$ & $\mathbf{A 5}$ \\
\hline $\mathrm{A} 1$ & 1 & $/$ & $/$ & $/$ & $/$ \\
\hline $\mathrm{A} 2$ & $0.511^{*}$ & 1 & $/$ & $/$ & $/$ \\
\hline $\mathrm{A} 3$ & $0.618^{* *}$ & $0.659^{* *}$ & 1 & $/$ & $/$ \\
\hline $\mathrm{A} 4$ & $0.905^{* * *}$ & $0.579^{*}$ & $0.759^{* *}$ & 1 & $/$ \\
\hline $\mathrm{A} 5$ & $0.585^{*}$ & $0.828^{* * *}$ & $0.716^{* *}$ & $0.779^{* *}$ & 1 \\
\hline
\end{tabular}

Table 6. Difference test on the overall construction quality of a teacher development school at different stages

\begin{tabular}{|l|c|c|c|c|}
\hline & Before construction & After construction & Mean difference & $\begin{array}{c}\text { Significance level } \\
\text { P-value }\end{array}$ \\
\hline Overall quality & 132.66 & 157.95 & -74.71 & 0.25 \\
\hline
\end{tabular}

Table 6 shows the difference test on the overall construction quality of a teacher development school at different stages. It can be found that the overall construction quality of the teacher development school is significantly better after construction than before construction, and it is considered that the difference is significant at the level of 0.25 . It indicates that under the guidance of periodical scores, the construction of a teacher development school can achieve significant progress. 


\section{$4 \quad$ Prediction of the Construction Quality of Teacher Development Schools}

Based on an improved artificial neural network, this paper constructed a predictive model for evaluating the construction quality of teacher development schools. Since the index data for the construction quality evaluation of teacher development schools do not have data sample labels, and there are no historical data, the training samples are quite limited. Therefore, the unsupervised neural network is more suitable for evaluation and prediction. This paper combined the unsupervised self-organizing feature map neural network, which has a strong generalization ability, with the supervised BP neural network, to improve the convergence speed of the training errors and the accuracy of the network evaluation to an ideal level.

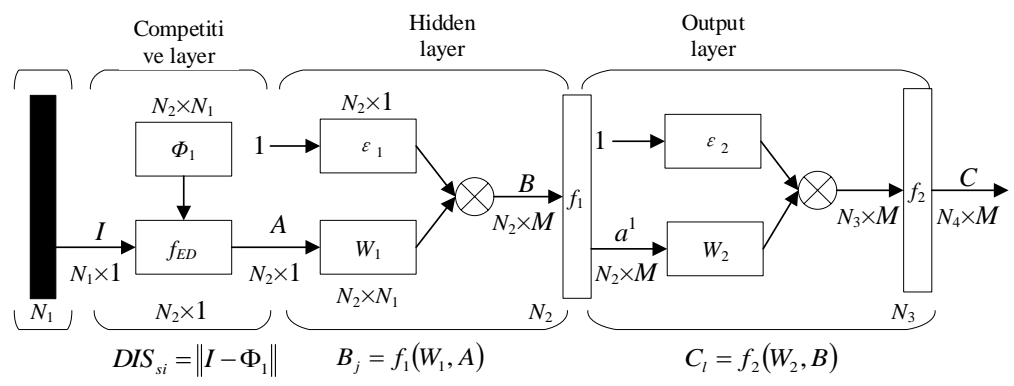

Fig. 1. Topological structure of the combined neural network

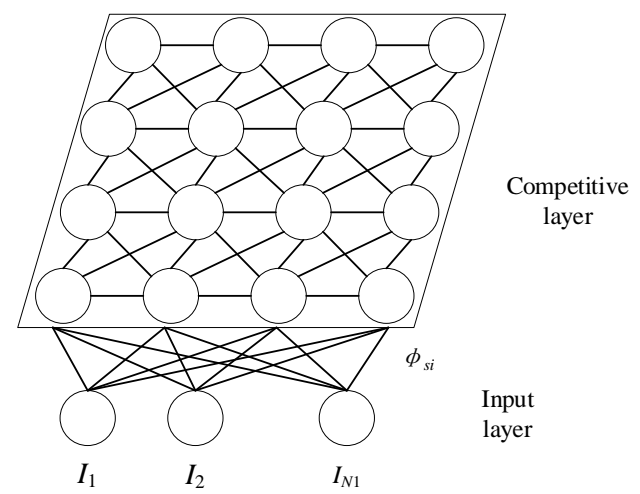

Fig. 2. Topological structure of the self-organizing feature map neural network

Figure 1 shows the basic structure of the constructed combined neural network, which consists of four layers, namely the input layer, the competitive layer, the hidden layer and the output layer. It can be seen that the training result of the self-organizing feature map neural network will be input as the input vector into the hidden layer of the BP neural network. In the figure, $N_{1}, N_{2}, N_{3}$ and $N_{4}$ are the number of neuron nodes in each layer, $\Phi_{1}, W_{1}$ and $W_{2}$ are the connection weights between the layers, $f_{E D}, f_{1}$ and $f_{2}$ 
are the excitation function of each layer, and $\varepsilon_{1}$ and $\varepsilon_{2}$ are the thresholds corresponding to the hidden layer and the output layer, respectively.

To complete evaluation prediction, the self-organizing feature map neural network requires multiple neurons in the competitive layer to cooperate, which avoids the situation where a single neuron is used to make evaluation decisions. Figure 2 shows the architecture of the self-organizing feature map neural network used in this paper.

Take $M$ index data samples for construction quality evaluation of a teacher development school as the input to the network, which are denoted as $I_{1}, I_{2}, \ldots, I_{N 1}$, and let the connection weight of the $i$-th neuron in the input layer to the $j$-th neuron in the competitive layer be $\varphi_{s i}$. To train the self-organizing feature map neural network, random values in the range of $[0,1]$ are first assigned to $\varphi_{s i}$ and the learning rate $\lambda(0)$. At the same time, the initial domain value $R_{k}(0)$ and the maximum number of iterations $T$ of the $k$ th competing neuron are also determined.

The Euclidean distance between the $s$-th neuron in the input layer and the $i$-th neuron in the competitive layer is calculated by Formula (1):

$$
D I S_{s i}=f_{E D}=\left\|I-\Phi_{1}\right\|=\sqrt{\sum_{s=1}^{N_{1}}\left[I_{s}(t)-\varphi_{s i}(t)\right]^{2}}
$$

The winning neuron is determined based on the distance calculated by Formula (1) - the neuron with the smallest Euclidean distance is the winning one, which matches best with the network input $I$ and satisfies the equation shown in Formula (2):

$$
P(I)=\arg \min \left\|I-\varphi_{s i}\right\|
$$

Suppose the update coefficient is $\delta$, then the connection weights of neurons in the domain can be updated through Formula (3):

$$
\varphi_{s i}(t+1)=\varphi_{s i}(t)+\delta \lambda(t)\left[I_{s}(t)-\varphi_{s i}(t)\right]
$$

The update rule of $\delta$ is shown in Formula (4):

$$
\delta=\left\{\begin{array}{l}
1 \quad i=k \\
0.5 \quad i \in R_{k}(t) \\
0 \quad \text { Otherwise }
\end{array}\right.
$$

The network learning rate $\lambda(t)$ in Formula (3) tends to gradually decrease linearly to 0 as the number of iterations $t$ increases. It can be calculated by Formula (5):

$$
\lambda(t)=K_{1} e^{\frac{-K_{2} t}{T}}
$$

where, $K_{1}$ and $K_{2}$ satisfy $0 \leqq K_{1} \leqq 1$ and $0 \leqq K_{2}$, respectively. Finally, judge the number of training times. If it reaches the set value, the training ends; otherwise, the training continues. Through continuous iterations, the Euclidean distance calculated by Formula (1) will become smaller and smaller until it converges, and finally the input samples 
belonging to the same winning neuron are classified into the same class. After network training, the connection weights between the output layer and the competitive layer of the network are determined.

The training of the selected three-layer BP neural network consists of two parts: the forward propagation of information from the input layer to the output layer and the back propagation of training errors for adjustment of the connection weights and thresholds. To train the BP neural network, parameters like the network training error $E$ and the learning rate $\alpha$ need to be initialized. The training results of the self-organizing feature map neural network are used as the input to the network, which are denoted as $A_{1}, A_{2}$, $\ldots, A_{N 2}$. The actual output of the network in the $t$-th iteration is set as $C_{1}^{t}, C_{2}^{t}, \ldots, C_{N 4}^{t}$, and the expected output is set as $D_{1}, D_{2}, \ldots D_{N 4}$.

Suppose the connection weight of the $i$-th neuron in the competitive layer to the $j$-th neuron in the hidden layer and the connection weight of the $j$-th neuron in the hidden layer to the $l$-th neuron in the output layer in the $t$-th iteration are respectively set as $\omega_{i j}{ }^{t}$ and $\omega_{j l}{ }^{t}$. The corresponding weight matrix is shown in Formula (6):

$$
W_{i j}^{t}=\left[\begin{array}{cccc}
\omega_{01}^{t} & \omega_{02}^{t} & \cdots & \omega_{0 j}^{t} \\
\omega_{11}^{t} & \omega_{12}^{t} & \cdots & \omega_{1 j}^{t} \\
\cdots & \cdots & \cdots & \cdots \\
\omega_{i 1}^{t} & \omega_{i 2}^{t} & \cdots & \omega_{i j}^{t}
\end{array}\right], W_{j l}^{t}=\left[\begin{array}{cccc}
\omega_{01}^{t} & \omega_{02}^{t} & \cdots & \omega_{0 l}^{t} \\
\omega_{11}^{t} & \omega_{12}^{t} & \cdots & \omega_{1 l}^{t} \\
\cdots & \cdots & \cdots & \cdots \\
\omega_{j 1}^{t} & \omega_{j 2}^{t} & \cdots & \omega_{j l}^{t}
\end{array}\right]
$$

After the network is initialized, values are randomly assigned to the initial network connection weight matrices $W_{i j}{ }^{0}$ and $W_{j k}{ }^{0}$. The numbers of neurons in the input layer and output layer of the neural network are determined by the dimensions $N_{2}$ and $N_{4}$ of the input samples and output evaluation results, and the number of nodes in the hidden layer is calculated by the empirical formula shown in Formula (7):

$$
N_{3}=\sqrt{N_{2}+N_{4}}+\beta
$$

where, $\beta$ is any constant greater than 1 and less than 10 . Then the input and output data are normalized, and the data limited between -1 and 1 and between 0 and 1 can be directly used for network training.

In the forward propagation of information, the output of the competitive layer enters the hidden layer as the input. Suppose the activation function of the hidden layer is $f_{1}$, then its output can be calculated by Formula (8):

$$
B_{j}=f_{1}\left(W_{1}, A\right)
$$

Suppose the activation function of the hidden layer is $f_{2}$, then its output can be calculated by Formula (9):

$$
C_{l}=f_{2}\left(W_{2}, B\right)
$$


According to the actual output and expected output, calculate the training error $E^{t}$ in the $t$-th iteration using Formula (10), and then determine whether it is less than the set error threshold $\varepsilon$. If it is so, the iteration ends; otherwise, the iteration continues.

$$
E^{t}=\frac{1}{2} \sum_{m=1}^{M} \sum_{l=1}^{N_{3}}\left(D_{m, l}-C_{m, l}\right)^{2}
$$

Then determine whether the training error in the $t+1$-th iteration meets the requirement. If it does, the iteration ends; otherwise, calculate the local gradients of the neurons in each layer of the network as follows:

$$
\left\{\begin{array}{c}
\nabla \gamma_{l}^{N_{3}}=\left(D_{m, l}-C_{m, l}^{t}\right)\left(1-C_{m, l}^{t}\right) C_{m, l}^{t} \\
\nabla \gamma_{j}^{N_{2}}=\left(\sum \nabla \gamma_{l}^{N_{3}} \omega_{j l}\right)\left(1-B_{j}\right) B_{j}
\end{array}\right.
$$

The network connection weights from the input layer to the hidden layer and the hidden layer to the output layer are updated through Formula (12):

$$
\left\{\begin{array}{l}
\omega_{j l}^{t+1}=\omega_{j l}^{t}+\alpha \nabla \gamma_{l}^{N_{3}} B_{j} \\
\omega_{i j}^{t+1}=\omega_{i j}^{t}+\alpha \nabla \gamma_{j}^{N_{2}} A_{i}
\end{array}\right.
$$

To train the combined neural network, it is necessary to normalize the training samples $I_{1}, I_{2}, \ldots, I_{N 1}$ and $W_{1}$. of the construction quality evaluation index data. For the competitive layer, based on the normalized results $\hat{A}$ and $\hat{W}_{1}$, calculate the Euclidean distance shown in Formula (13) and take the minimum value using Formula (12) to obtain the winning neuron.

$$
\begin{aligned}
& D I S^{\prime}=\min \left\|\hat{A}_{i}-\hat{W}_{1}\right\| \\
& =\min \sqrt{2\left(I-\hat{\omega}_{1, i j} \cdot \hat{A}_{i}\right)}
\end{aligned}
$$




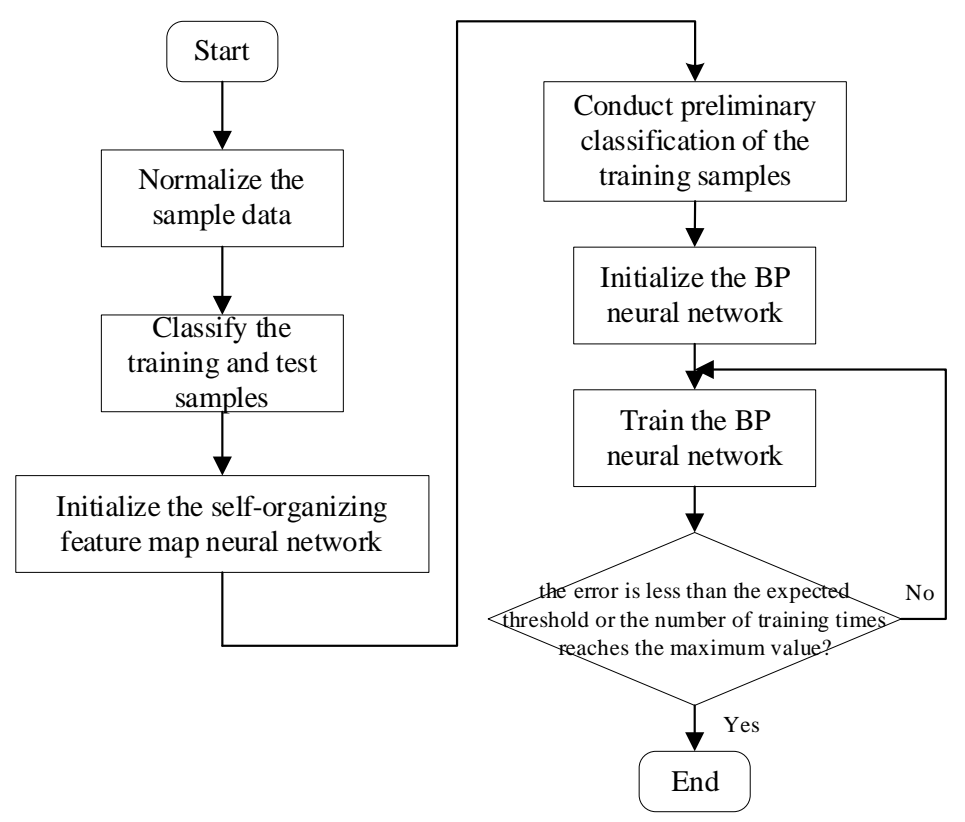

Fig. 3. Flow chart of the combined neural network algorithm

The winning neuron obtained through Formula (13) has the right to adjust its weight vector $W_{1}$. The algorithm flow is shown in Figure 3, and the specific steps are described as follows:

1. Normalize the index data samples for teacher development school construction quality evaluation and classify the training samples and test samples.

2. Initialize the self-organizing feature map neural network in the combined network, and perform preliminary classification of the training samples.

3. Initialize the BP neural network in the combined network, and use the classified results as the input for further network training.

4. Determine whether the training error is less than the set threshold and whether the number of iterations is smaller than the set maximum number of iterations. If either condition is satisfied, the training ends; otherwise, continue to adjust and update the connection weights and thresholds of the BP neural network until either of the conditions is met.

\section{$5 \quad$ Experimental Results and Analysis}

In the Matlab simulation environment, this paper took the construction quality evaluation of a teacher development school as an example to verify the effectiveness of the combined neural network (combining the BP neural network and the self-organizing feature map neural network) in quality evaluation. The combined network retains the unique advantages of the two networks - the self-organizing feature map neural network 
can perform preliminary classification of the construction quality data of the teacher development school when there are a small amount of sample data, which can effectively reduce the load of the BP neural network in data processing, while the BP neural network makes up for the shortcoming of the self-organizing feature map neural network - lack of classification accuracy, and is responsible for the main quality evaluation tasks.

This paper selected 30 groups from 200 groups of construction quality evaluation data samples of a teacher development school as the test samples, and the remaining 170 groups as the training ones. First, the principal component analysis method was used to perform front-end data processing of the training samples, and then the processed data samples were used as the input to train the combined network. Finally, four quality ratings were set, namely excellent, good, medium and poor.

Figure 4 shows the training error curves of the BP neural network and the combined neural network. Unlike in the BP neural network, where the training error reached the preset value after about 100 iterations, the training error in the combined neural network converged to 0.0127 only after 26 iterations, which proves that the combined neural network has a faster convergence speed.

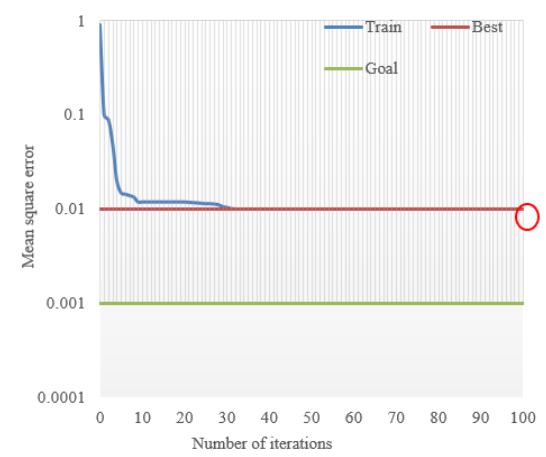

a)

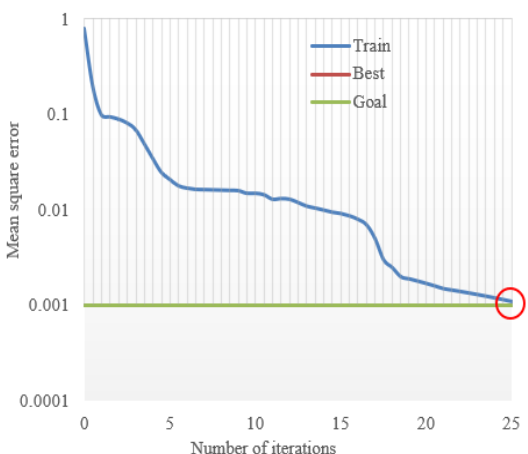

b)

Fig. 4. Training error curves before and after the application of the combined neural network

Table 7. Neural network training results before and after the application of the combined neural network

\begin{tabular}{|l|c|c|c|}
\hline \multicolumn{1}{|c|}{ Network type } & Training error & Number of iterations & Convergence \\
\hline Self-organizing feature map neural network & 0.1024 & 125 & Converged \\
\hline BP neural network & 0.0554 & 110 & Converged \\
\hline Combined neural network & 0.00143 & 25 & Converged \\
\hline
\end{tabular}

Table 7 gives a more visual data display. It can be seen that, the training error of the combined neural network already converged to the preset target error in the $26^{\text {th }}$ 
iteration, while those in the self-organizing feature map neural network and the BP neural network needed 125 and 110 times of training respectively to converge.

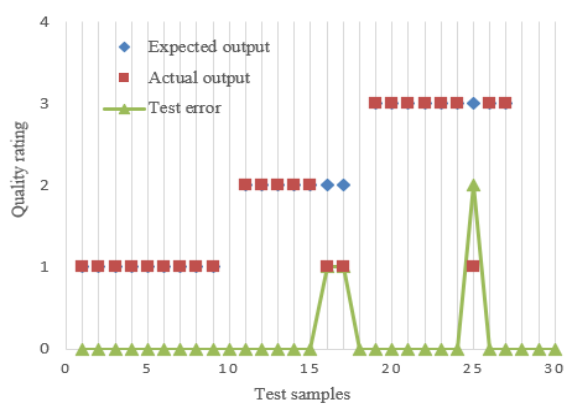

a)

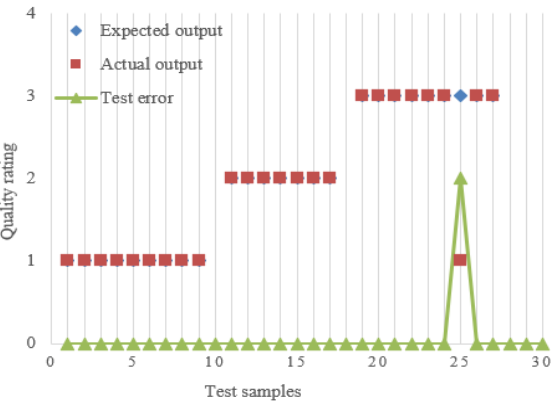

b)

Fig. 5. Quality rating errors before and after the application of the combined neural network

For ease of observation, Figure 5 shows a comparison of the actual predicted results and the expected output results of quality ratings. The blue diamonds represent the expected output, the red squares the actual predicted results and the green triangles the absolute errors, i.e. the deviations of the predicted results from the expected output. It can be seen that the combined neural network had a recognition rate of $100 \%$ for the construction quality evaluation index data of the teacher development school. Compared with the BP neural network, which had 3 classification errors in the classification of 30 sample groups (and thus the classification accuracy was $90 \%$ ), the combined neural network had 1 classification error (and thus the classification accuracy was 96.7\%). The quality evaluation results were basically consistent with the actual ones, thus proving that the model is feasible for evaluating the construction status of teacher development schools. Figure 6 compares the quality evaluation accuracy before and after the application of the combined network, and it can be clearly seen that the combined neural network has a higher accuracy than either of the two networks alone, indicating that the combined neural network structure can help improve the evaluation accuracy. 


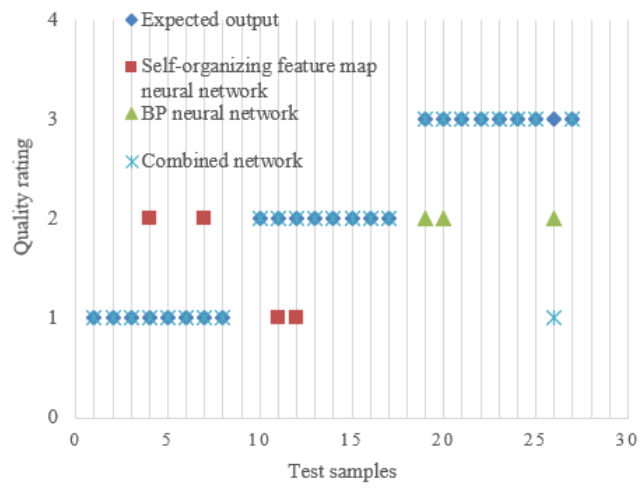

Fig. 6. Accuracy of quality evaluation before and after the application of the combined network

\section{Conclusion}

In order to accurately evaluate the construction quality of teacher development schools through actual case analysis, this paper proposed a method for evaluating the construction quality of teacher development schools based on an improved artificial neural network. First, this paper constructed an evaluation index system for the construction quality of teacher development schools consisting of five core evaluation indices, namely learning group, training quality density, organizational control function, fairness and process management, and then established detailed periodical scoring criteria for the 20 evaluation sub-indices. After that, it performed reliability, validity and difference analysis of the evaluation system for the construction quality of teacher development schools to verify its feasibility. Finally, it constructed an improved neural network combining the self-organizing feature map neural network and the BP neural network to predict the construction quality ratings of teacher development schools. The experimental results proved the effective prediction function of the model from the perspectives of network convergence, data recognition rate and evaluation accuracy.

\section{$7 \quad$ Acknowledgement}

This work was supported by the Second Batch of Teaching Reform Program during the 13th Five-Year Plan Period of Higher Education of Zhejiang Province of China (No. jg20190690).

\section{$8 \quad$ References}

[1] Harmon, E., Hotton, V., Liebman, R., Lupro, M., Feng, W.C., Delcambre, L., Pouliot, D. (2020). Cyberpdx: An interdisciplinary professional development program for middle and 
high school teachers. Annual Conference on Innovation and Technology in Computer Science Education, ITiCSE, 1335. https://doi.org/10.1145/3328778.3372652

[2] Laborda, J.G., Díaz, V.C., Ramírez, E.J. (2020). Foreign Language Pre-Service Teachers' Attitudes Towards Integrated Technology, International Journal of Emerging Technologies in Learning, 15(23), 85-94. https://doi.org/10.3991/ijet.v15i23.18797

[3] Stevenson, M., Bower, M., Falloon, G., Forbes, A., Hatzigianni, M. (2019). By design: Professional learning ecologies to develop primary school teachers' makerspaces pedagogical capabilities. British Journal of Educational Technology, 50(3): 1260-1274. https://doi.org/10.1111/bjet.12743

[4] Mouza, C., Barrett-Greenly, T. (2015). Bridging the app gap: An examination of a professional development initiative on mobile learning in urban schools. Computers and Education, 88: 1-14. https://doi.org/10.1016/j.compedu.2015.04.009

[5] Zhao, J.Y. (2010). School knowledge management framework and strategies: The new perspective on teacher professional development. Computers in Human Behavior, 26(2): 168-175. https://doi.org/10.1016/j.chb.2009.10.009

[6] Al-Mubireek, S. (2020). Teacher Perceptions of the Effectiveness of Using Handheld Devices in Saudi EFL Classroom Practices, International Journal of Emerging Technologies in Learning, 15(22), 204-217. https://doi.org/10.3991/ijet.v15i22.16689

[7] Iqbal, H.M., Shayer, M. (2000). Accelerating the development of formal thinking in Pakistan secondary school students: achievement effects and professional development issues. Journal of Research in Science Teaching, 37(3): 259-274. https://doi.org/10.1002/(sici) 1098-2736(200003)37:3<259::aid-tea3>3.0.co;2-w

[8] Altinay, F., Beyatli, Ö., Dagli, G., Altinay, Z. (2020). The role of Edmodo model for professional development: The uses of blockchain in school management. International Journal of Emerging Technologies in Learning, 15(12): 256-270. https://www.learntechlib. org/p/217556/. https://doi.org/10.3991/ijet.v15i12.13571

[9] Loudova, I. (2020). Continuing Professional Development in ICT for Primary School Teachers, Reflections and Issues. Lecture Notes in Computer Science (including subseries Lecture Notes in Artificial Intelligence and Lecture Notes in Bioinformatics), 12218: 326337. https://doi.org/10.1007/978-3-030-51968-1_27

[10] Sayabek, Z., Arzayeva, M., Gulbazar, M., Mustafina, A., Ulan, T. (2017). Key issues in relation to leading and managing educational change: Professional development in secondary school. Proceedings of the 30th International Business Information Management Association Conference, IBIMA 2017 - Vision 2020: Sustainable Economic development, Innovation Management, and Global Growth, 4152-4160.

[11] Johnson, C.C., Kahle, J.B., Fargo, J.D. (2007). A study of the effect of sustained, wholeschool professional development on student achievement in science. Journal of Research in Science Teaching, 44(6): 775-786. https://doi.org/10.1002/tea.20149

[12] Annetta, L.A., Shymansky, J.A. (2006). Investigating science learning for rural elementary school teachers in a professional-development project through three distance-education strategies. Journal of Research in Science Teaching, 43(10): 1019-1039. https://doi.org/ $10.1002 /$ tea.20106

[13] Blasie, C., Palladino, G. (2005). Implementing the professional development standards: A research department's innovative masters degree program for high school chemistry teachers. Journal of Chemical Education, 82(4): 567-570. https://doi.org/10.1021/ ed082p567

[14] Hixon, E., Buckenmeyer, J. (2009). Revisiting technology integration in Schools: Implications for professional development. Computers in the Schools, 26(2): 130-146. https://doi.org/10.1080/07380560902906070 
[15] Sweeney, A.E., Bula, O.A., Cornett, J.W. (2001). The role of personal practice theories in the professional development of a beginning high school chemistry teacher. Journal of Research in Science Teaching, 38(4): 408-441. https://doi.org/10.1002/tea.1012

[16] Mitchell, J.T., Roy, G., Fritch, S., Wood, B. (2018). GIS professional development for teachers: lessons learned from high-needs schools. Cartography and Geographic Information Science, 45(4): 292-304. https://doi.org/10.1080/15230406.2017.1421482

[17] Lin, C.D., Lin, M.J., Zhang, X.S. (2011). Can we transform the e-teacher community to be a learning organization to promote school teachers' professional development. 2011 IEEE 3rd International Conference on Communication Software and Networks, ICCSN 2011, 245-249. https://doi.org/10.1109/iccsn.2011.6013819

[18] Oostendorp, A., Buijnsters, P. Peeters, V., Van Lieshout, H., Aarts, J. (1996). The development of learning materials for medical informatics education in Dutch professional schools of health care. Studies in Health Technology and Informatics, 34: 828-832.

[19] Khalid, S., Strange, M.H. (2016). School teacher professional development in online communities of practice: A systematic literature review. Proceedings of the European Conference on e-Learning, ECEL, 605-614.

[20] Holley, D., Howlett, P. (2016). Engaging our school teachers: An augmented reality (AR) approach to continuous professional development. Lecture Notes of the Institute for Computer Sciences, Social-Informatics and Telecommunications Engineering, LNICST, 160, 118-125. https://doi.org/10.1007/978-3-319-28883-3_15

[21] Belova, S., Travkina, N., Podchalimov, M., Kretova, V. (2015). Creation of conditions for professional retraining and professional development of employees of the city construction organizations in Kursk Assembly Technical School. Procedia Engineering, 117(1): 80-84. https://doi.org/10.1016/j.proeng.2015.08.127

[22] Goode, J., Peterson, K., Malyn-Smith, J., Chapman, G. (2020). Online Professional Development for High School Computer Science Teachers: Features That Support an Equity-Based Professional Learning Community. Computing in Science and Engineering, 22(5): 51-59. 10.1109/MCSE.2020.2989622 https://doi.org/10.1109/mcse.2020.2989622

[23] Yang, R., Porter, A.C., Massey, C.M., Merlino, J.F., Desimone, L.M. (2020). Curriculumbased teacher professional development in middle school science: A comparison of training focused on cognitive science principles versus content knowledge. Journal of Research in Science Teaching, 57(4): 536-566. https://doi.org/10.1002/tea.21605

\section{$9 \quad$ Author}

Xiaoyu Hua was born in Ningbo, Zhejiang, Associate Professor of Ningbo Childhood Education college, her research interests include Educational Technology and Education Management (Email: 2000005@ncec.edu.cn)

Article submitted 2021-02-23. Resubmitted 2021-04-02. Final acceptance 2021-04-04. Final version published as submitted by the authors. 